\title{
DER MENSCH ALS GEOLOGISCHER FAKTOR
}

\author{
HEINRICH JÄCKLI
}

\section{JAHRE KANDERDURCHSTICH}

Am 12. November des vergangenen Jahres waren es 250 Jahre her, seit die Kander, der herrliche Bergfluß des Berner Oberlandes, durch einen künstlich geschaffenen Kanal quer durch den Strättligen-Hügel fließt und sich in den Thunersee ergießt. Bis dahin, nämlich bis zum 12. November 1713, war sie durch das jetzige Glütschbachtälchen geflossen und hatte die Aare auf der Höhe der Zulgmündung erreicht. Kander und Zulg hatten immer wieder die Talsohle von Thun bis gegen Uttigen mit ihren Geschiebemassen überführt und dabei gleichzeitig einen Rückstau der geschiebefreien Aare und häufige Uferüberflutungen dem Thunersee entlang verursacht. Die Anwohner hatten allerdings lange diese geologischen Verhältnisse als weitgehend unveränderliche Gegebenheiten empfunden und sich ihnen angepaßt, die Siedlungen den überschwemmungssicheren Flanken der Ebene entlang angelegt, ebenso auch die Verkehrswege, und anderseits die während den Überschwemmungen zum Sedimentationsgebiet gewordene Talsohle nach Möglichkeit gemieden.

Und dann beschloß der Große Rat von Bern, als geologischer Faktor zu wirken, den Strättligen-Hügel zu durchstechen und die Hochwasser der Kander auf direktestem Wege in den Thunersee einzuleiten (Fig. 1). Anfänglich war diese Flußableitung, die erste ihrer Art in der Schweiz, mit einem Stollen erstellt worden, über welchen die wichtige Landstraße von Bern ins Oberland führte. Dem neuen Flußbett zum Thunersee wagte man, unbeschwert von allzu großen wasserbaulichen und geologischen Fachkenntnissen, ein Gefälle von $6 \frac{1}{2} \%$ zu geben. Als sich aber dann im Sommer 1714 das erste Hochwasser der Kander einstellte, begann der Fluß in dieser kurzen, zu steilen Strecke in Schottern und Moränenlehm intensiv zu erodieren; die rückschreitende Erosion führte zur Absenkung des Flußbettes flußaufwärts, schließlich zur vollständigen Trockenlegung des früheren Kanderbettes im Glütschtal, zum Einsturz des Tunnels und zu einer grundlegenden Veränderung jenes Landschaftsbildes: alles Ereignisse, die man im Programm keineswegs vorgesehen hatte. Bis 1890 hatte die Bettvertiefung bis zur Vereinigung von Simme und Kander, also rund $3 \mathrm{~km}$ oberhalb der Mündung in den See, volle $21 \mathrm{~m}$ erreicht!

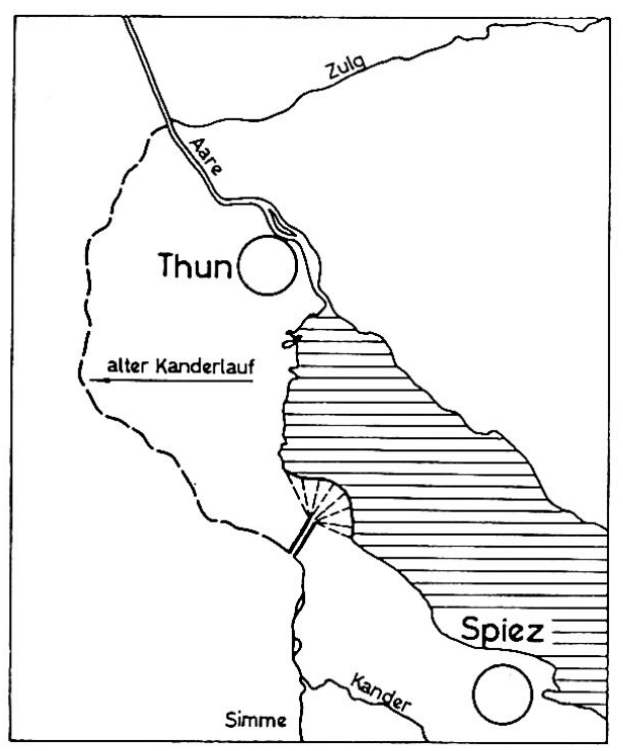

Figur 1

Die Ableitung der Kander in den Thunersee $(1: 111000) 1711-1713$.

Ursprünglich war sie nur als Hochwasserableitung gedacht, doch wurde im Sommer 1714 das alte Kanderbett infolge Tiefenerosion unfreiwillig trockengelegt 


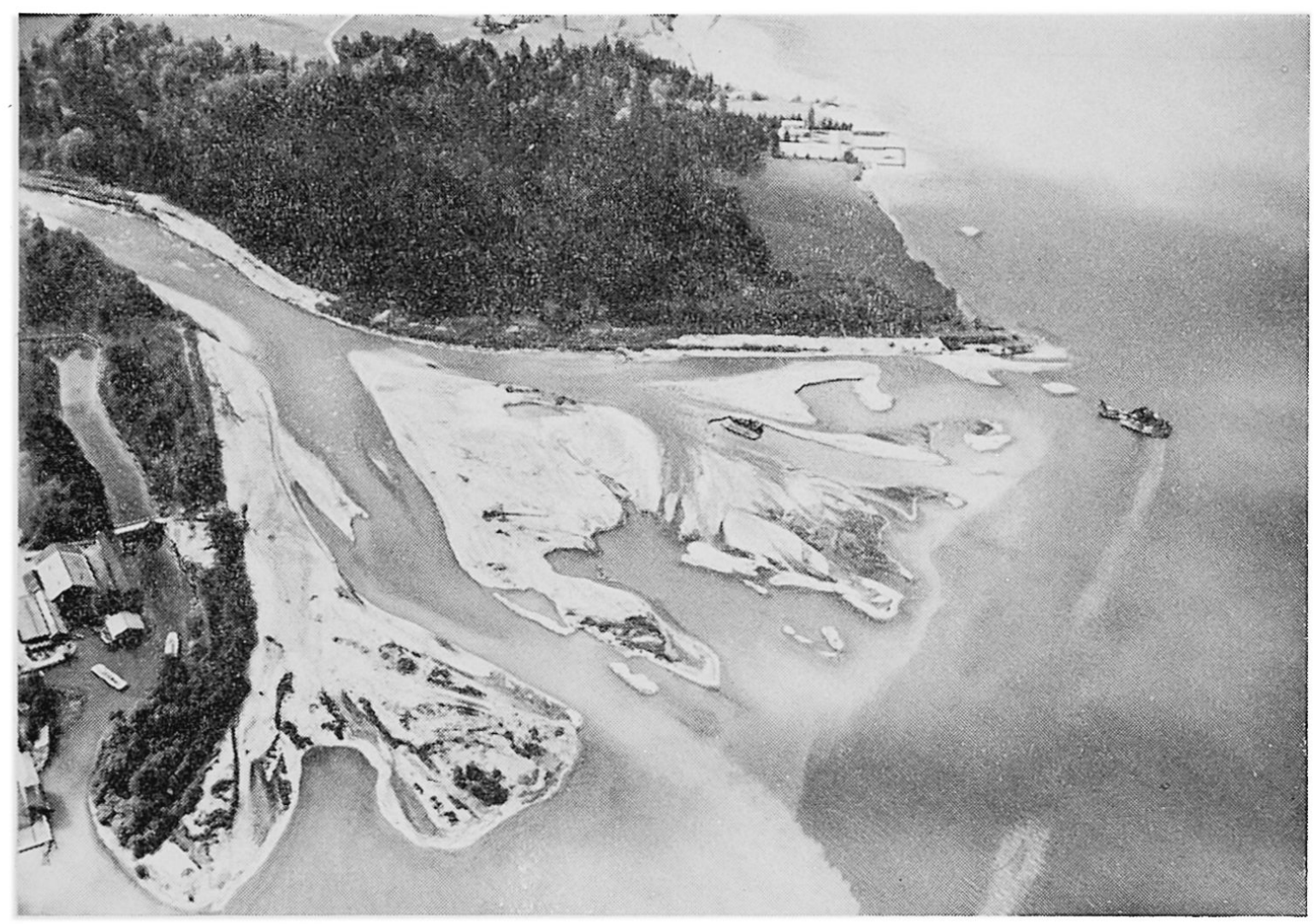

Figur 2 Luftbild des Kanderdeltas im Thunersee

Das ganze sichtbare Gelände ist in den letzten 250 Jahren entstanden. Die auf dem Bilde sichtbaren Schwimmbagger entfernen laufend einen wesentlichen Teil des anfallenden Kieses, wodurch das Deltawachstum künstlich verlangsamt wird.

Aufnahme: Swissair Photo AG

Am Thunerseeufer entstand ein neues Flußdelta, das dank der Schuttzufuhr aus dem alpinen Einzugsgebiet relativ rasch anwuchs (Fig. 2). Auf Grund des natürlichen Wachstums dieses Deltas, dessen 250. Geburtstag wir jetzt feiern, läßt sich rückwärts ausrechnen, daß die Kander im Mittel pro Jahr rund $300000 \mathrm{~m}^{3}$ Gesteinsmaterial im Thunersee ablagerte oder pro $\mathrm{km}^{2}$ Einzugsgebiet rund $363 \mathrm{~m}^{3}$. Bei einem Raumgewicht von rund 2,0 t/m $/ \mathrm{m}^{3}$ entspricht das einem Abtrag, gleichmäßig auf das Einzugsgebiet verteilt, von $0,27 \mathrm{~mm}$ pro Jahr. Setzt man voraus, der Abtrag sei proportional zur mittleren Höhe des Einzugsgebietes über der Erosionsbasis, dem Thunersee, so beträgt die «Halbwertzeit des Abtrages», die in diesem Fall nötig wäre, um die mittlere Höhe des Einzugsgebietes auf die Hälfte zu reduzieren, rund 3,35 Millionen Jahre, vorausgesetzt allerdings, daß die Erdkruste sich nicht verstellt, das heutige Klima sich nicht verändert und - der Mensch nicht eingreift.

Der «Kanderdurchstich» und die 1871-1877 durchgeführte Korrektion der Aare von Thun bis Uttigen ermöglichten es, das einstige Überschwemmungsgebiet der Talsohle unterhalb des Thunersees in fruchtbares Land und bevorzugtes Wohngebiet zu verwandeln. Dieses Beispiel möge zeigen, daß der Mensch primär und von jeher sich nicht nur der Natur anzupassen, sondern ebensosehr seine Umgebung und selbst geologische Vorgänge seinen Bedürfnissen entsprechend umzugestalten versucht.

Im folgenden sei überprüft, wo und wieweit er sich rein passiv geologischen Gegebenheiten anpaßt und wo er anderseits aktiv auf geologische Vorgänge einen Einfluß ausübt, sei es bewußt, um solche geologischen Prozesse zu verändern, sei es ungewollt, indem er durchaus unerwünschte Veränderungen unfreiwillig auslöst. 


\section{ANPASSUNG AN GEOLOGISCHE GEGEBENHEITEN}

Der Mensch meidet Gebiete geologischer Aktivität. Er meidet Sedimentationsgebiete, die sich durch immer wiederkehrende Überschwemmungen auszeichnen, wie auch Erosionsgebiete, bei denen Rutschungen oder Wildbachschäden aller Art zu erwarten sind. Für seine Siedlungen und Verkehrswege sucht er Gebiete geringster geologischer Aktivität, Fels- und Moränenkuppen, Terrassen über der tiefsten Talsohle, tote Schuttkegel.

Die moderne Ingenieurgeologie stellt die wissenschaftliche Methode dar, sich den geologischen Verhältnissen anzupassen. Wo eine Staumauer plaziert werden kann, wo ein Laufwerk sich eignet, wo ein dichter Stauraum für einen Jahresspeicher geschaffen werden kann, wo man mit einem Stollen einer Rutschung ausweichen muß, wie tief der Grundwasserspiegel liegt, das abzuklären ist Aufgabe des Geologen, um dem Ingenieur zu zeigen, wie er für seine Kunstbauten die geologischen Gegebenheiten berücksichtigen kann.

$\mathrm{Daß}$ aber die richtige Einschätzung dieser Gegebenheiten und damit die zweckmäßigste Anpassung an sie auch heute noch auf Schwierigkeiten stößt oder sogar mißlingt, haben die Katastrophen von Malpasset und von Vaiont auf tragische Weise demonstriert :

Am 2. Dezember 1959 brach die Staumaucr Malpasset am Reyran oberhalb Fréjus im Departement Var ein, der dahinter erstmals voll aufgestaute Stausee entleerte sich schlagartig und die Fluten zerstörten dabei Teile der an der Küste gelegenen Stadt Fréjus. Die Untersuchung nach der Katastrophe hat dann gezeigt, da $B$ nicht etwa der Beton, sondern vielmehr der Fels seitlich und unterhalb der Betonmauer dem Wasserdruck nicht standgehalten hat; längs einer tektonisch angelegten Gleitfläche war der Fels unmittelbar unter dem linken Widerlager abgeschert worden. Der Gneis war deutlich verwittert und offensichtlich nicht in der Lage gewesen, der hohen Beanspruchung bei gefülltem Stausee standzuhalten. Man hatte geglaubt, ohne tiefgründige Injektionen auszukommen, die Qualität des Felsens nicht künstlich verbessern zu müssen, und hatte sich in dieser Beziehung im Fels getäuscht.

In der Nacht des 9. Oktober 1963 stürzte eine Gesteinsmasse von rund $300 \mathrm{Mio} \mathrm{m}^{3}$ seitlich in den halbvollen künstlichen Stausee von Vaiont im Piavetal in Oberitalien, verursachte dadurch eine einmalige riesige Flutwelle, die sich über die Staumauer ergoß, ohne sie zum Einsturz zu bringen, die jedoch flußabwärts katastrophale Verheerungen anrichtete und gegen 3000 Tote forderte. Die eingesetzten Experten haben nun nachträglich abklären müssen, wieweit durch die für Jahresspeicher üblichen starken Schwankungen des Seespiegels eine ohnehin labile Felsflanke in beschleunigte Bewegung versetzt wurde, wieweit der Umfang und die Geschwindigkeit dieser Rutschbewegung unterschätzt und ihre Wirkung auf den See falsch berechnet und ob Evakuationsempfehlungen oder -befehle nicht weitergegeben oder zu wenig ernst genommen worden sind.

\section{BEWUSSTE GEOLOGISCHE VERÄNDERUNGEN}

$\mathrm{Zu}$ den ältesten Beeinflussungen geologischer Vorgänge durch den Menschen gehören zweifellos die flußbaulichen Eingriffe, konzentrierten sich doch fast alle Hochkulturen des Altertums auf Alluvialebenen längs wichtiger Flüsse, die vorerst kanalisiert und gebändigt werden mußten, bevor ihre Umgebung bewohnbar wurde. Aus der Schweiz wurde bereits eingangs der «Kanderdurchstich» in den Thunersee erwähnt; eine klassische Arbeit analoger Art ist das «Linthwerk» (Fig. 3), als 1811 die Linth am Ausgang aus dem Glarnerland künstlich in den Walensee eingeleitet wurde, oder 1878 die Einleitung der Aare in den Bielersee im Zuge der ersten Juragewässerkorrektion (Fig. 4). In allen diesen drei Fällen wies der Mensch dem Fluß ein neues Sedimentationsgebiet am Seeufer zu, wo nun an gewünschter Stelle der Fluß ein neues Delta aufbaut, während gleichzeitig das vorher von Überschwemmungen heimgesuchte Alluvialgebiet trockengelegt wurde und nun landwirtschaftlich genutzt und besiedelt werden kann.

Einen interessanten Eingriff in die Sedimentationsverhältnisse bildet die künstliche Kolmatierung der Talsohle des Domleschg in Graubünden mit Schlamm des Nolla, des berüchtigten, bei Thusis aus dem Beveringebiet in den Rhein mündenden Wildbaches. 


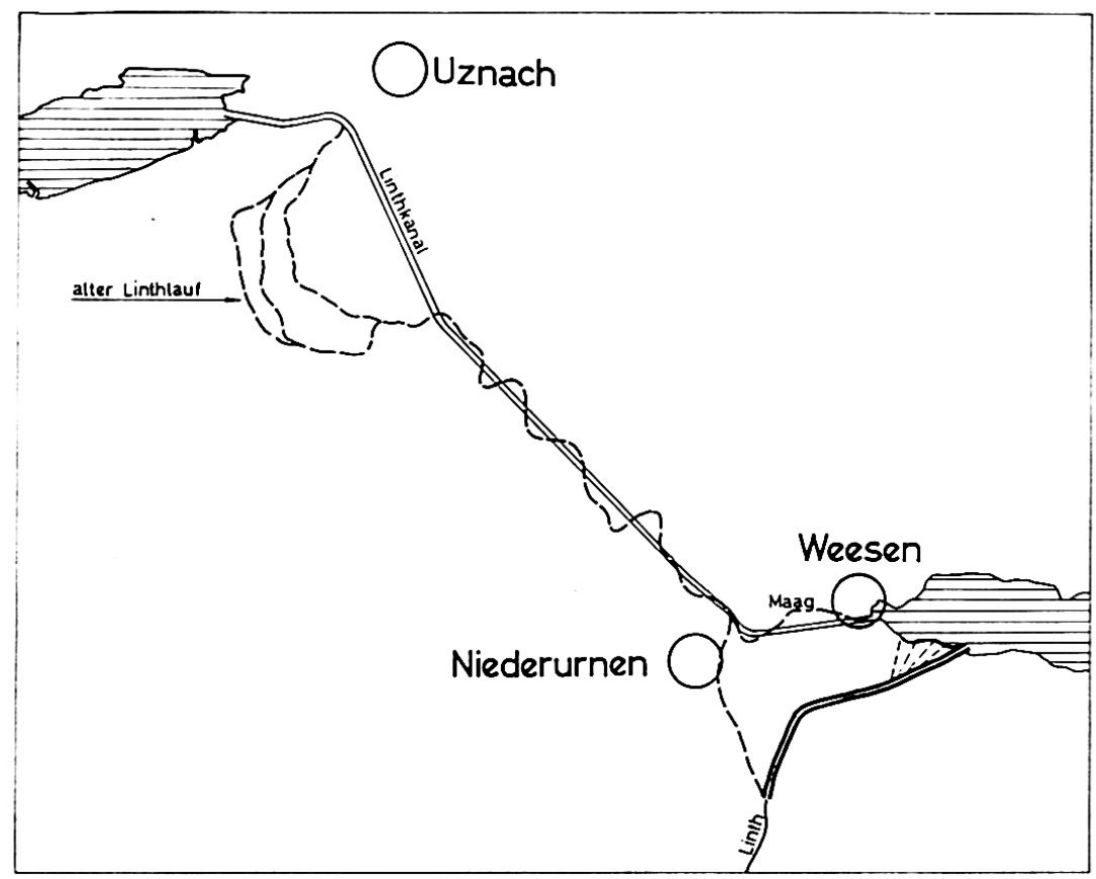

Figur 3

Die Ableitung der Linth in den Walensee $(1: 160000)$ 1805-1811, im Rahmen des * Linthwerkes * unter Hans Konrad Escher ausgeführt

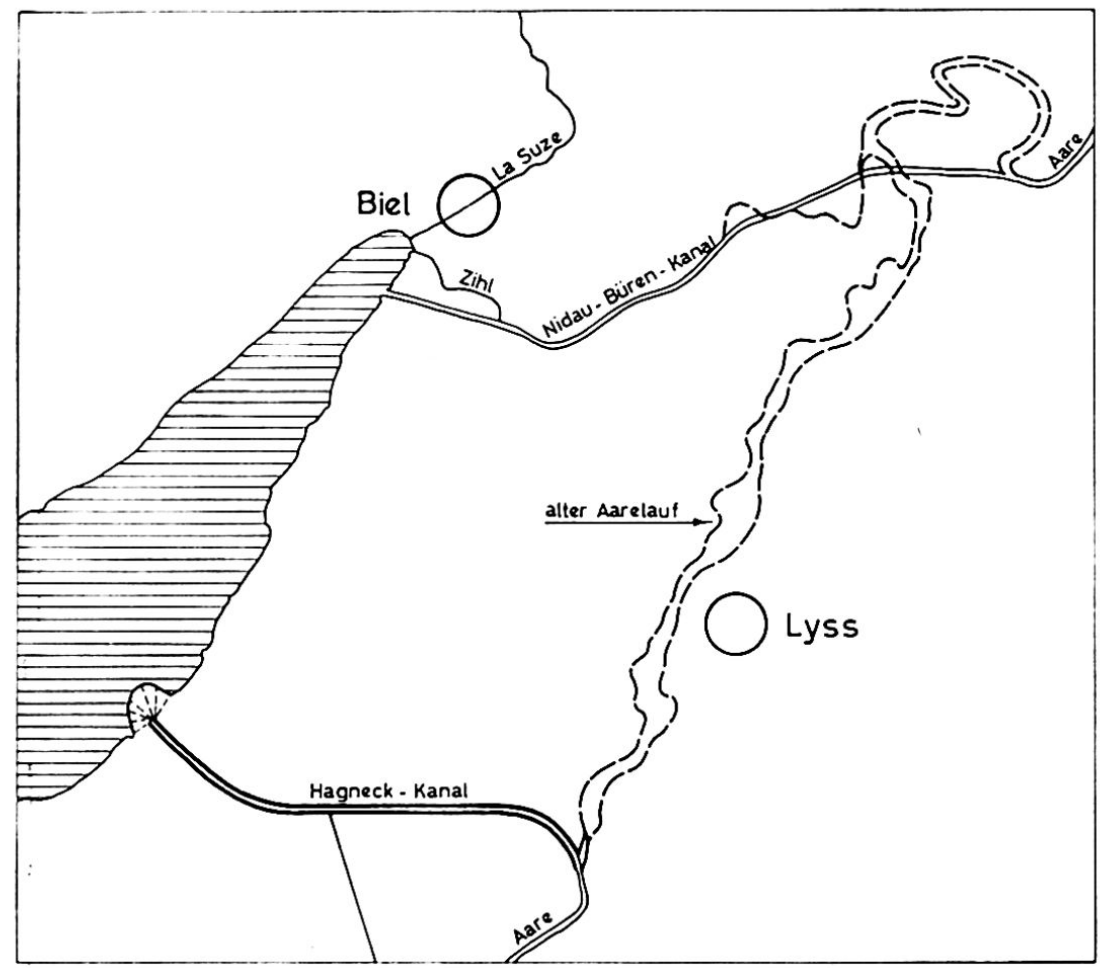

Figur 4

Die Ableitung der Aare in den Bielersee (1:160000) 1868-1878 im Zuge der ersten Juragewässerkorrektion ausgeführt

Die vorher unfruchtbare Kiesebene wird systematisch Feld für Feld mittels künstlicher Überflutung mit rund $80 \mathrm{~cm}$ nährstoffreichem tonigem Bachschlamm bedeckt, so daß schließlich die kiesige Talsohle, die vorher kaum einen Ertrag abwarf, zu einem landwirtschaftlich wertvollen Gebiet werden wird.

Wenn der Mensch dem Hauptfluß verbietet, in der Talsohle aufzuschottern, zu kolmatieren, indem er links und rechts des Flußbettes Dämme errichtet und den Fluß streckt, so muß dieser Fluß seinen Schutt an einem anderen Ort ablagern, beispielsweise an seiner Mündung, im Delta. Dadurch wächst ein solches Delta infolge der Kanalisierung des Oberlaufes rascher als im natürlichen Zustand, der Flußlauf wird dadurch 
rascher verlängert, und durch die Verlängerung verlangt das Flußbett eine raschere Erhöhung als im natürlichen Zustand. Diesen Fall finden wir heute in der Poebene, wo der Po seinen Schutt aus den Alpen erst im Delta an der Adriaküste deponieren kann, während die Dämme ständig erhöht werden müssen und ein Ende dieser Dammerhöhungen nicht abzusehen ist. Eine seitliche Kolmatierung wäre zweifellos geologisch wirkungsvoller und auch dem natürlichen Geschehen viel entsprechender; sie läßt sich im Falle der Poebene nachträglich aber nicht mehr nachholen, weil die Talsohle unterdessen mit Siedlungen und Verkehrswegen belegt wurde.

Mittels Wildbachverbauungen versucht der Mensch bewußt, die Tiefenerosion zu verlangsamen, indem er Quersperren ins Bachbett einbaut. Üblicherweise reagiert dann der Bach mit Seitenerosion. Will man auch diese unterbinden, so ist man gezwungen, die einzelnen Quersperren mit Leitwerken zu verbinden.

Aber diese Maßnahmen zur Geschiebeverminderung im Einzugsgebiet, im Oberlauf, haben im Unterlauf des Gewässers zur Folge, daß dort der gegenüber früher nun geschiebeärmer gewordene Fluß eine verstärkte Tendenz zur Erosion zeigt und daß das häufig recht labile Gleichgewicht zwischen Aufschotterung und Eintiefung zugunsten der letzteren umschlägt, indem der Fluß vor den Augen der erstaunten Anwohner zu erodieren beginnt, wo er sich früher im Gleichgewicht befand.

Vom Ausmaß der künstlichen Eingriffe längs Meeresküsten, wie sie in großzügigster Weise etwa in Holland mit der Trockenlegung der Zuidersee oder im «Delta-Plan» an die Hand genommen wurden, vermögen wir Binnenländer uns nur mit Mühe eine Vorstellung zu machen.

\section{NICHT GEWOLLTE, ABER GEDULDETE GEOLOGISCHE VERÄNDERUNGEN}

Als Baugruben, als Stollen und Kavernen, als Steinbrüche und Kiesgruben schafft der Mensch Hohlformen, deren Material er für irgendwelche $Z$ wecke verwendet, ohne daß er bewußt geologische Veränderungen verursachen möchte. Als künstliche Deponien schafft er umgekehrt neue Vollformen, indem er das Ausbruchmaterial irgendwo schüttet als neuen Berg, als neue Halde, als neuen Damm, weil er sonst nicht weiß, wohin damit.

In Stauseen schaffen wir künstliche Sedimentationsräume, wo früher Erosion geherrscht hatte, und lassen dort limnische Sedimente zur Ablagerung gelangen, statt dieses Material durch den Fluß talabwärts transportieren zu lassen. Durch solche Stauseen wird einerseits das Abflußregime des Flusses verändert, meist im Sinne einer Brechung der Spitzen und damit einer Reduktion der Transport- und Erosionskraft, anderseits wird aber auch das Transportregime des Flusses verändert, indem er geschiebefrei aus dem Stausee austritt und dementsprechend im Delta in der Regel weniger Material ablagert als früher, vorausgesetzt, $d a ß$ der Stauraum nicht periodisch gespült wird.

\section{UNGEWOLLTE, NICHT VORAUSGESEHENE VERÄNDERUNGEN}

Es gibt Eingriffe des Menschen in das geologische Geschehen, die durchaus ungewollt sind, die vom Ménschen nicht beherrscht werden und bei denen er in der Regel erstaunt ist, daß seine Eingriffe diese Folgen gehabt haben. Er spielt bei solchen Vorgängen die unrühmliche Rolle des Zauberlehrlings.

$\mathrm{Zu}$ solchen ungewollten Veränderungen gehört die nicht vorausgesehene Tiefenerosion der Kander beim Durchstich in den Thunersee. Kiesbaggerungen und Streckung und Eindämmung von Flüssen können zu Tiefenerosion und als deren Folge zu Uferrutschungen und zur Gefährdung von Brückenfundamenten führen. Es gehören dazu alle die künstlich ausgelösten Bergstürze, wie jener von Plurs am 25. August 1618 oder von Elm am 11. September 1881, in beiden Fällen durch unsachgemäßen Abbau von Bausteinen verursacht. 
In früheren Jahrhunderten führten die Entwaldungen durch Kahlschläge zu verstärkter Erosion und zur Bildung von Wildbächen; im letzten und in diesem Jahrhundert verursachte die einseitige landwirtschaftliche Ausnützung von Lockerböden die berüchtigten «Badlands», jene ihrer Humusschicht entblößten, feinziselierten Erosionsstrukturen. Durch den Weidgang war schon im Altertum und im Mittelalter das Mittelmeergebiet dermaßen entwaldet und seiner schützenden Grasnarbe beraubt worden, daß seither trotz großen Aufwendungen die Neubildung von Wald und geschlossenem Wiesland als bester Schutz gegen Erosion erst bescheidene Fortschritte gemacht hat.

Die zunehmende Eutrophierung unserer Seen verursacht ungewollt einen grundlegenden Fazieswechsel in den rezenten Seesedimenten, mit einem Umschlag von sauerstoffreicher weißer Seekreide zu schwarzem, sauerstofflosem Faulschlamm, der am Grunde des Zürichsees 1898 einsetzte und seither in den tiefsten Sedimenten vieler anderer Seebecken ebenfalls beobachtet wurde.

Die Belastung unserer Flüsse mit organischem Schmutz beeinträchtigt in Infiltrationsgebieten die Qualität des Grundwassers.

Starke künstliche Grundwasserabsenkungen, Bergbau oder Salzauslaugungen verursachen an der Oberfläche ungleichmäßige Terrainsenkungen.

\section{RÜCKWIRKUNGEN AUF DAS PRINZIP DES AKTUALISMUS}

Das Prinzip des Aktualismus als Forschungsmethode wurde vor rund 150 Jahren in die Geologie eingeführt, erstmals von K. E. A. von Hoff, dann von Ch. Lyell und anderen. Eine konsequente Anwendung des Aktualismus stellt beispielsweise die zeitliche Extrapolation der heutigen Abtragsvorgänge, wie wir sie aus den Deltavermessungen rekonstruieren können, auf Vergangenheit und Zukunft dar.

Der Mensch bringt nun einen anthropogenen Störungsfaktor ins natürliche geologische Geschehen, der ein an-aktualistisches Moment darstellt, das früher fehlte und das in Zukunft progressiv zunehmen wird. Der menschliche Einfluß fehlte früher vollständig, ist aber heute vielerorts bereits so stark, daß gemessene Vorgänge nicht mehr ohne weiteres rückwärts extrapoliert, d. h. nicht vorbehaltlos aktualistisch im ursprünglichen, klassischen Sinn ausgewertet werden dürfen.

Um beim Deltawachstum unserer Alpenflüsse zu bleiben: Beschleunigend wirken Waldrodungen und Kahlschläge, verzögernd dagegen Aufforstungen, Hangentwässerungen, Wildbachverbauungen, künstliche Kolmatierungen, künstliche Stauseen, Kiesbaggerungen im Flußbett und im Delta.

Auf die Meteorologie, die ihrerseits bekanntlich den ausschlaggebendsten Faktor in der exogenen Geologie darstellt, hat der Mensch bis vor kurzem noch keinen wesentlichen Einfluß nehmen können. Ob dem so bleiben wird, bezweifeln wir aber ernsthaft. Wir leben heute vorderhand noch in der «vor-anthropometeorologischen» Aera, um die uns möglicherweise unsere Nachfahren einst beneiden werden.

In der Kette der nie abreißenden Naturvorgänge spielte der Faktor Mensch neben allen übrigen geologischen Faktoren bisher eine örtlich beschränkte und zeitlich erst seit 2-3000 Jahren wirkende Rolle. Wenn wir jedoch in Zukunft mit nuklearen Sprengstoffen Bergbau betreiben, mit nuklearen Mitteln Schiffahrtswege bauen und Flüsse ableiten werden, um nur einige nichtmilitärische Aspekte zu nennen, wird sich das bisherige Verhältnis allerdings grundlegend ändern.

Unsere Generation hat die Verantwortung gegenüber der uns anvertrauten und uns gewissermaßen ausgelieferten Erde zu tragen, was verlangen würde, daß der Mensch als geologischer Faktor mit Überlegung und mit $\mathrm{Maß}$ wirkt und nur solche geologischen Veränderungen einleitet, deren Wirkungen und Folgen er tatsächlich zu überblicken vermag. 


\title{
LITERATURVERZEICHNIS
}

Beck, P. (1943): Die Natur des Amtes Thun. Bülow, K. von (1954): An-aktualistische Wesenszüge der Gegenwart. Zschr. Deutsch. Geol. Ges. Bd. 105. Collet, L. W. (1916) : Le charriage des alluvions. Annalen der schweiz. Landeshydrographie. Bern. Dittmer, E. (1955): Der Mensch als geologischer Faktor an der Nordseeküste. «Eiszeitalter und Gegenwart». Häusler, H. (1959) : Das Wirken des Menschen im geologischen Geschehen. Naturk. Jb. der Stadt Linz. Jäckli, H. (1957): Gegenwartsgeologie des bündnerischen Rheingebietes. Ein Beitrag zur exogenen Dynamik alpiner Gebirgslandschaften. Beitr. Geol. d. Schweiz, geotechn. Serie, Lieferung 36; - (1958) : Der rezente Abtrag der Alpen im Spiegel der Vorlandsedimentation. Eclogae geol. Helv., Vol. 51, No. 2 (Basel). Steck, Th. (1892) : Die Denudation im Kandergebiet. J. br. geogr. Ges. Bern, Bd. XI. Vogt, J. (1958) : Zur historischen Bodenerosion in Mitteldeutschland. Peterm. Mitt. 3.

\section{L'HOMME COMME FACTEUR GÉOLOGIQUE}

Suivant le cas et suivant sa volonté l'homme cherche à excercer son influence dans les processus géologiques, par exemple en dérivant artificiellement un fleuve dans un lac. Souvenons-nous de la dérivation de la Kander dans le lac de Thoune, il y a 250 ans; ce fut la première grande intervention de l'homme dans les processus naturels de la sédimentation d'un fleuve alpin suisse. En outre l'homme provoque beaucoup d'autres transformations, par exemple par des travaux artificiels d'excavation et de remblaiement dans les régions minières, etc. Enfin, l'homme provoque également des processus géologiques involontaires et incontrôlés comme par exemple: l'éboulement de Plurs et d'Elm dû à l'exploitation d'une carrière, ou les inondations de Fréjus et de Vaiont causées par la présence d'un barrage.

De cette manière l'homme provoque aujourd'hui le dérèglement des processus géologiques, ce qui ne se produisait pas dans le passé, mais qui augmentera progressivement dans l'avenir.

\section{DUBROVNIK}

\author{
HERBERT GOTTSCHALK
}

Mit Farbbild

Sehnsucht fast aller Dalmatien-Reisenden ist Dubrovnik, die «Perle an der Adria», oft auch «das slawische Athen» genannt. Diese schöne Stadt bezaubert durch den Glanz der südlichen Natur, das malerische Weiß ihrer Mauern und Türme, die aus dem schimmernden Blau des Meeres steigen. All diese Vorzüge bieten eine reizvolle Kulisse für den Fremdenverkehr, auf den hier alles abgestimmt ist. Im Trubel der Saison geht daher leicht die tiefere Sicht in die Seele der Menschen verloren, die sich in der Abgeschiedenheit der Inseln noch unverfälscht öffnet. Doch wenn im Frühling oder Herbst Mauern, Gassen und Treppen noch nicht von vielsprachiger Geschwätzigkeit erfüllt sind, wenn der Schirokko übers Meer bläst, dann öffnet sich leichter die Atmosphäre einer ruhmvollen Vergangenheit.

Die Geschichte der Republik Dubrovnik ist so alt wie die seiner jahrhundertelangen Rivalin Venedig. Den Anfang machte ein römisches Kastell, in das sich die Bewohner der griechischen Siedlung Epidaurus vor den Awaren flüchteten. Dadurch wurde eine Erweiterung des Befestigungsgürtels nötig, und bald hatte Dubrovnik innerhalb des oströmischen Reiches eine eigene Gesetzgebung erreicht. Tapfer erwehrte sich die kleine Stadt der Seeräuber mit Hilfe des sagenhaften Roland, dessen 1418 geschaffene Statue noch heute vor der barocken St-Blasius-Kirche steht. Die Seeräuber waren in jener Zeit so mächtig, daß selbst Venedig ihnen eine direkte Steuer zahlte, um seinen Schiffen freie Durchfahrt zu sichern. Die aufblühende Stadt Ragusa, wie Dubrovnik noch bis vor kurzer Zeit hieß, war zeitweise an Byzanz und auch an die Bane der Herzegowina tributpflichtig, aber ihr Streben war schon am Anfang auf Selbständigkeit gerichtet. Durch ein Bündnis mit den sizilianischen Normannen gewann sie wichtige Handels- 